\title{
Nutlet morphology and its taxonomic implication in some taxa of Lamiaceae in Egypt
}

\author{
Wafaa M. Kamel \\ Botany Department, Faculty of Science, Suez Canal University, Ismailia- \\ Egypt
}

Wafaa M. Kamel, 2014. Nutlet morphology and its taxonomic implication in some taxa of Lamiaceae in Egypt. Taeckholmia 34: 101-127.

\begin{abstract}
$N_{\text {utlet morphology of } 28 \text { taxa belonging to } 15 \text { genera of Lamiaceae }}$ were examined using light and scanning electron microscopy. The taxa included representatives of the three subfamilies Ajugoideae, Lamioideae and Nepetoideae which all collected especially from Sinai, Egypt. Morphological characters, including nutlet shape, color, size; hilum (shape, length $\mathrm{x}$ width and position); epidermal cell shape; anticlinal walls (level, undulation, thickness and secondary sculpture); outer periclinal cell walls (level and secondary sculpture) are presented. The reticulate type was the most common among the studied taxa. Four types of basic anticlinal cell wall boundaries were recognized and many different shapes of the outer periclinal cell wall were described. Nutlet ornamentation, shape, size, colour and particularly hilum characters proved to be good taxonomic characters for the Egyptian Lamiaceae taxa. A key for the identification of the investigated taxa based on nutlet characters is provided.
\end{abstract}

Key words: Lamiaceae, nutlet morphology, SEM, Egypt.

\section{Introduction}

Lamiaceae (Labiatae) is one of the largest angiospermic families, includes 236 genera and about 7173 species. They are almost cosmopolitan but absent from the coldest regions of high altitude or latitude, including many well-known plants, herbs, shrubs and trees of horticultural, economic and medicinal significance (Harley et al. 2004). Hedge (1992) recognized six regions of high Labiatae diversity. The Mediterranean and SW Central

Received 17 Oct. 2014, Accepted 10 Nov. 2014 
Asiatic regions represent one of the greatest centers of diversity of many genera.

In the flora of Egypt, Lamiaceae is the tenth largest plant family, represented by 23 genera and 55 species, four of which are endemic in Egypt (Boulos 2002, 2009). Täckholm (1974) reported 22 genera and 56 species. El-Husseini (1986) in her Ph.D. Thesis recorded also 22 genera and only 54 species, whereas El-Hadidi and Fayed (1995) reported only 20 genera and 51 species. Based on the most recent classification of Harley et al. (2004) and Bendiksby et al. (2011), Lamiaceae is divided into seven subfamilies. In this study all Egyptian Lamiaceae taxa are classified under the three subfamilies Ajugoideae, Lamioideae and Nepetoideae (Harley et al. 2004 and Bendiksby et al. 2011).

Heywood (1971) drew attention to the importance and impact of scanning electron microscopy (SEM) in the study of the systematic problems, as very valuable information has been provided by using this technique.

Nutlet morphology and micromrphology in Lamiaceae has proved useful to varying degrees at different levels of the taxonomic hierarchy, depending on the characters chosen and variation present. Studies on nutlet morphology and pericarp structure in Lamiaceae are increasing (Cantino 1990 and 1992; Husain et al. 1990; Demissew and Harley 1992; Ryding 1993, 1994 and 1998; Marin et al. 1994and Oran 1996). In recent years, the importance of scanning electron microscopy (SEM) in the study of nutlets surface and its taxonomic value has been ascertained for various genera of Lamiaceae (Stace 1989; Husain et al. 1990; Stuessy 1990; Ryding 1993; Marin et al. 1994, 1996; Oran 1996; Guerin 2005, Salmaki et al. 2008; Hassan et al. 2012; Mousavi et al. 2013and Tarimcilar et al. 2013). These studies showed that nutlet characters are potentially useful within the Lamiaceae at the level of section, genus and species.

Ryding (1993, 1994) studied nutlet characters in Leucas and related genera of subfamily Lamioideae and pericarp structure and phylogeny of subfamily Podostemonoideae of Lamiaceae using SEM. He found that nutlet surfaces were typically smooth,while sub-surface characters were used to distinguish taxa. Marin et al. (1994) characterized nutlets of Teucrium L. using the presence and density of oil glands. Oran (1996) found that gross nutlet morphology and surface sculpturing in species of Salvia L. was variable and taxonomically useful. He developed descriptive categories 
for shape, surface sculpturing pattern and cellular deposits. Husain et al. (1990) studied the micromorphology in the tribe Saturejeae and found that sculpturing patterns (most commonly reticulate) were the most useful characters. Demissew and Harley (1992) studied the seed epidermis and stated that surface types correlated with the three infrageneric groups of Stachys in tropical Africa. The surfaces types recognized were coarsely reticulate, finely reticulate, and reticulate- spinulose. Guerin (2005) studied nutlets of Hemigenia and Microcorys. Significant variation, mainly useful at the infrageneric level, was found in nutlet shape, nature of the attachment scar, nature of surface sculpturing, exocarp cell shape and sculpturing, and nature of the indumentums. Salmaki et al. (2008) studied nutlets micromorphology and its systematic implication in 31 taxa of Stachys, representing nine sections and one species of a closely genus distributed in Iran. However, the type of sculpturing is more useful for separating species within the section, rather than correlating the related ones to each other. Hassan et al. (2012) studied nutlets micromorphology of thirteen species of Nepeta. Two main patterns, reticulate and indistict reticulate were described. Mousavi et al. (2013), studied micromorphology features of ten Salvia nutlets growing in NE Iran. They divided these species into three and four groups based on the shape and ornamentation of nutlets respectively. The variation in color, size and ornamentation of mericarp helped to identify species. Tarimcilar et al. (2013) investigated nutlet morphology of 11 taxa of Mentha distributed throughout Turkey using SEM. In their study, they divided Mentha into three groups, based on nutlet sculpturing type such as distinctly bireticulate, inconspicuously bireticulate and reticulate.

Husain et al. (1990) concluded that features of nutlets were useful in providing evidence for phylogenetic reconstruction. Cantino (1992) and Ryding (1998) used nutlet characters, including ultrastructure, surface morphology and shape, in cladistic analyses within Lamiaceae, but they did not elaborate on the nature or selection of the characters.

Although there are many studies on the nutlet characters of family Lamiaceae as shown above, only few Egyptian species have been treated e.g. Teucrium polium ( Marin et al. 1994); Salvia sclarea ( Marin et al. 1996 and Mousavi et al. 2013); Salvia palaestina ( Kahraman and Dogan 2010) and Mentha longifolia ( Tarimcilar et al. 2013). Therefore the present investigations deal with morphological characters of nutlet (mericarp) of 28 taxa belonging to 15 genera of Lamiaceae in Egypt, especially in Sinai, to 
find good nutlet characters for comparison and delimitation of genera and species in the family.

\section{Materials and Methods}

This study is based on mature nutlets of 28 species of Lamiaceae. Sample of each taxon were taken from field collections and herbarium specimens ( Table 1). Voucher specimens are deposited in the Herbarium of the Suez Canal University, Faculty of Science, Ismailia, Egypt (SCU-I, proposed acronym of the herbarium). The collected specimens were identified according to Täckholm (1974) and Boulos (2002, 2009).

The external macromorphological aspects of the nutlets of the studied taxa were examined by light stereomicroscope. At least 10-15 mature nutlets for each taxon were taken to cover the range of variations. For scanning electron microscopy ( SEM) investigation, the nutlets were dried and fixed to specimens stubs with an adhesive material and placed on the revoloving discs of Joel fine coat ion sputter ( Joel, JFC 1100E). Each nutlet was uniformly coated with a thin layer of gold, then scanned and photographed using JEOL JSM 5300 SEM with an accelerating voltage of $15 \mathrm{kv}$ at Electron Microscopic Unit, Faculty of Science, Assiut University (Egypt). Actually, nutlets were studied from different views, but when the sculpturing patterns varied at different views, the dorsal surfaces were chosen for description. Nutlet surface sculpturing terminology follows Barthlott (1981) and Stearn (1983). Selected SEM micrographs of nutlets are presented in Figs. (1-33).

The main features of the investigated nutlets are summarized in Table $(2,3$ $\& 4)$. The shape of nutlets showed types of variation among the investigated taxa (Figs. 1-33).

\section{Result and Discussion}

The main features of the investigated nutlets are summarized in Table (2, $3 \& 4)$. The shape of nutlets showed types of variation among the investigated taxa (Figs. 1-33).

Nutlet shape varies from obovoid (e.g. Ajuga iva and Stachys aegyptiaca), ellipsoid (Teucrium leucocladum), ellipsoid-obovoid (Teucrium decaisnei), obovoid-trigonous (Phlomis aurea), oblong (Marrubium alysson and Lavandula coronopifolia), oblanceoloid-ellipsoid 
(Salvia deserti), subglobose (Mentha longifolia subsp. typhoides), globose (Salvia palaestina and S. spinosa) to ovoid-oblong (Thymus decussatus).

Nutlet size ranged between 0.6-5.5 mm in length and $0.2-2.5 \mathrm{~mm}$ in width. Among the examined taxa Phlomis aurea has the largest nutlets (4.3$5.5 \times 1.9-2.5 \mathrm{~mm})$ followed by Salvia palaestina $(3.2-3.7 \times 2.5-3 \mathrm{~mm})$, while species like Mentha longifolia subsp. typhoides $(0.6-0.7 \times 0.4-0.5 \mathrm{~mm})$ and Micromeria sinaica possessed small $(0.7-1$ x $0.2-0.3 \mathrm{~mm})$ nutlets (Table 2). Most of the studied taxa showed glabrous nutlets (23 taxa), but the remaining five species covered by sparsely or dense sessile glandular hairs e.g. Ballota undulata, Marrubium alysson, M. vulgare, Lavandula coronopifolia and L. pubescens.

Nutlet colour were ranging from pale brown or brown (Lavandula pubescens), brown (Marrubium vulgare, Thymus bovei and T. decussatus), dark brown (Teucrium decaisnei and T.polium), yellowish brown (Micromeria sinaica), orange brown (Origanum syriacum subsp. sinaicum ), greyish to black (Ajuga iva), dark brown-black (Stachys aegyptiaca and Nepeta septemcrenata) and black (Salvia aegyptiaca, S. deserti and $S$. lanigera ) ; except for Lamium amplexicaule dark brown with irregular whitish spots; and Salvia multicaulis, S. palaestina, S. sclarea and S. spinosa have pale or dark brown with darker net veins. Luster of nutlets surface varies from matte in 14 taxa, semi matte in 12 taxa and glossy in Salvia palaestina and S. spinosa (Table 2).

Dorsal surface of nutlets showed three types of variation among the investigated taxa. Most nutlets were convex (24 taxa); more or less convex in Otostegia fruticosa and Micromeria nervosa and convex-trigonous in Lavandula coronopifolia and L. pubescens. The ventral mid rib of the nutlet was indistinct in 20 taxa; distinct in the three species Lamium amplexicaule, Otostegia fruticosa and Phlomis aurea. Moreover four taxa have distinct midrib toward the base e.g. Marrubium alysson, M. vulgare,Stachys aegyptiaca and Mentha longifolia subsp. typhoides. Ballota undulata was the only species having a more or less distinct mid rib.The majority of the nutlets apex were rounded (23 taxa). The apex was rounded with more or less mucronate tip in one taxon (Stachys aegyptiaca); truncate in 3 species, Lamium amplexicaule, Otostegia fruticosa and Phlomis aurea and obtuse in Micromeria sinaica (Table 2).

Base of nutlet was rounded in 10 taxa and obtuse in 7 taxa . Lamium amplexicaule was characterized by having a nutlet with an elongated, light 
coloured elaiosome cells at the truncate base. This result agrees with that given by Strasburger (1978), Wojciechowska(1972), and Packham(1983). Base was truncate in Ajuga chamaepitys subsp. tridactylites and Salvia sclarea; rounded with more or less pointed tip in Marrubium vulgare; obtuse with more or less small beak in Thymus decussates; shortly attenuate in Otostegia fruticosa and shortly attenuate-obtuse in the remaining 5 taxa.

Shape of hilum showed either obovoid (e.g. Ajuga chamaepitys subsp. tridactylites , A.iva, Thymus bovei and T. decussatus ), triangular (Lamium amplexicaule and Marrubium alysson), subglobose-globose (Teucrium polium, T. leucocladum), globose (Teucrium decaisnei), rhomboidalglobose (Salvia lanigera), discoid (Salvia multicaulis), bilobed (Nepta septemcrenata), ovoid (Micromeria nervosa), ellipsoid (Micromeria sinaica) or obcordate-rounded (Lavandula coronopifolia and L. pubescens). In some taxa hilum was present at lateral position others have basal and subbasal position, mostly without rim (22 taxa). Hilum size ranged from $0.06-1.9 \mathrm{~mm}$ in length and $0.05-1.3 \mathrm{~mm}$ in width. Among the examined taxa Ajuga chamaepitys subsp. tridactylites and A.iva showed the largest hilum (1.3-1.4 X 0.6- 0.7mm \& 1.8-1.9 X 1.2-1.3 mm), while Micromeria nervosa and M.sinaica possessed small (0.06-0.1 x 0.05-0.1mm \& 0.08-0.1 x $0.05-0.07 \mathrm{~mm}$ ) hilum. The two genera, Ajuga and Teucrium, showed significant length of the hilum (often over half length of nutlet). This result agree with that given by Guerin (2005) and Özkan et al. (2009). Since the shape, postion and size of hilum varies within the species, so it was useful character for delimitation of the taxa (Table 4).

Homogeneity of testa cell size of the studied taxa were variable from regular, more or less regular and irregular. It was regular in Teucrium decaisnei, T. leucocladum, Otostegia fruticosa, Mentha longifolia subsp.typhoides, Micromeria nervosa, M. sinaica, Thymus bovei and $T$. decussatus (figs. 3c,4c,10c,15c,16c,17c,27c,28c). Marrubium alysson is more or less regular, (fig.8c). The remaining taxa (19 taxa) had irregular testa cell size. Epidermal cell shape varies from tetra-penta-hexagonal to elongated or rounded.

Surface ornamentation: Regarding the nutlet surface ornamentation, four main types can be distinguished: I. reticulate (the most common type), II. rugose, III. ruminate, and IV. smooth slightly tuberculate. Within these types, variants were recognized (Table 3).

Type I: Reticulate: be divided into 8 subtypes. These are: 
Subtype 1: Reticulate, this sculpture pattern is recorded in Ajuga iva, Ballota undulata, Lamium amplexicaule, Stachys aegyptiaca, Lavandula pubescens, L. coronopifolia, Mentha longifolia subsp. typhoides, Micromeria nervosa, Salvia aegyptiaca, S.deserti and Thymus bovei (figs. 2c,6c,7c,12c,13c,14c, 15c,16c, 20c, 21c, 27c).

Subtype 2: reticulate to reticulate-colliculate in Teucrium decaisnei, T.leucocladum, T. polium (figs.3c, 4c, 5c).

Subtype 3: upper portion reticulate (1/3 length) and remaining portion transversely rugose (2/3 length) in Ajuga chamaepitys subsp. tridactylites (fig. 1c).

Subtype 4: reticulate-verrucate in Marrubium vulgare M. alysson, Salvia lanigera (figs. 8c, 9c, 22c).

Subtype 5: reticulate-ruminate in Otostegia fruticosa (fig.10c).

Subtype 6: reticulate-tuberculate in Nepeta septemcrenata (fig.18c). Subtype 7: reticulate-foveate in Salvia multicaulis and S. spinosa (figs. 23c, 26c).

Subtype 8: indistinct reticulate in Thymus decussatus (fig. 28c).

Type II: rugose: this type is divided into3 subtypes as follows:

Subtype 1: irregular rugose- scrobiculate in Phlomis aurea (fig. 11c).

Subtype 3: longitudal coarse rugose in Micromeria sinaica (fig.17c).

Subtype 4: sclariform-rugose in Salvia sclarea (fig.25c). This result agree with those given by Mousavi et al. 2013 and do not agree to Marin et al. 1996.

Type III: ruminate: in Origanum syriacum subsp. sinaicum (fig. 19c).

Type IV: smooth, slightly tuberculate in Salvia palaestina (fig. 24c). This result is similar to that given by Kahraman and Dogan 2010.

Anticlinal cell wall boundaries were generally well developed (Table 3). These were four types:

1. channelled in 5 taxa: Marrubium alysson, Stachys aegyptiaca, Micromeria nervosa, Thymus bovei and T.decussatus (figs. 8c, 12c, $16 \mathrm{c}, 27 \mathrm{c}, 28 \mathrm{c})$.

2. slightly raised in 7 taxa: Teucrium decaisnei, T. leucocladum, Lavandula pubescens, Mentha longifolia subsp.typhoides, Salvia palaestina, S.sclarea and S.spinosa (figs. 3c, 4c, 14c, 15c, 24c, 25c, 26c).

3. more or less raised in Phlomis aurea (fig. 11c). 
4. raised in the remaining 15 taxa (figs. 1c, 2c, 5c, 6c, 7c, 9c, 10c, 13c, $17 \mathrm{c}, 18 \mathrm{c}, 19 \mathrm{c}, 20 \mathrm{c}, 21 \mathrm{c}, 22 \mathrm{c}, 23 \mathrm{c})$. Undulation of the anticlinal walls were straight in most of the studied taxa (21); more or less straight in Ajuga chamaepitys subsp. tridactylites, Lamium amplexicaule and Salvia lanigera (figs. 1c, 7c, 22c); undulate in Ajuga iva, Origanum syriacum subsp. sinaicum, Salvia sclarea and Thymus decussatus (figs. 2c, 19c, 25c, 28c). The anticlinal walls were distinct thick in Ajuga iva and Mentha longifolia subsp.typhoides (figs. 2c, 15c); thick in 7 taxa Otostegia fruticosa, Marrubium vulgare, Micromeria sinaica, Origanum syriacum subsp. sinaicum, Salvia palaestina, S.sclarea and S. spinosa (figs.10c, 13c, 17c, 19c, 24c, 25c, 26c); more or less thick in Ballota undulata, Phlomis aurea and Salvia lanigera (figs $.6 c, 11 c, 22 c)$; and thin in the remaining taxa (16). The patterns of the secondary sculpture in anticlinal walls varies from greatly smooth in Salvia spinosa (fig.26c); undulate in Ajuga iva, Lamium amplexicaule and Origanum syriacum subsp. sinaicum (figs. 2c, 7c, 19c); ribbed in Micromeria sinaica and Salvia sclarea (figs. 17c, 25c) and striate in the 22 remaining taxa.

Periclinal walls were distinctly flat in Phlomis aurea, Mentha longifolia subsp.typhoides and Micromeria nervosa (figs. 11c, 15c, 16c); flat to slightly convex in Thymus decussatus (fig. 28c); flat to slightly concave in Marrubium alysson, Stachys aegyptiaca, Lavandula pubescens, Saliva sclarea and Thymus bovei (figs. 8c, 12c, 14c, 25c, 27c); concave with sunken center in Otostegia fruticosa and Lavandula coronopifolia (figs. 10c, 13c); slightly concave in Teucrium decaisnei, Origanum syriacum subsp. sinaicum, Salvia palaestina and S. spinosa (figs. 3c, 19c, 24c, 26c) and concave in the remaining 13 taxa.

Secondary sculpture (fine relief of cell wall) in periclinal cell walls showed also great variations. It varies from striate-favulariate e.g. Ajuga chamaepitys subsp. tridactylites,Teucrium decaisnei, Marrubium vulgare (Figs. 1c, 3c, 9c); smooth in Ajuga iva, Phlomis aurea, Micromeria sinaica, Origanum syriacum subsp. sinaicum and Salvia palaestina (Figs. 2c, 11c, 17c, 19c, 24c); favulariate in Teucrium leucocladum and Salvia lanigera (Figs. 4c, 22c); striate to micro-crimped papillae in Teucrium polium (Fig. $5 \mathrm{c}$ ); striate with mostly globular cental pappillae in Mentha longifolia (Fig. 15c); striate to micro-reticulate in Ballota undulata, Thymus bovei and T.decussatus (Figs. 6c, 27c, 28c); ruminate to micro-reticulate in Lamium 
amplexicaule ( Fig. 7c); smooth outer-striate in center in Marrubium alysson(Fig. 8c); striate-foveate in Otostegia fruticosa (Fig. 10c); striaterugose in Stachys aegyptiaca (Figs. 12c); striate in Lavandula coronopifolia; L. pubescens, Nepta septemcrenata, Salvia aegyptiaca, S. deserti and $S$. multiculais (Figs. 13c, 14c, 18c, 20c, 21c, 23c); ruminate in Salvia sclarea (Fig. 25c); foveate-smooth in Salvia spinosa (Fig. 26c) and circular striate in Micromeria nervosa (Fig. 16c).

\section{Key to the studied taxa based on Nutlet characters}

1a. Nutlets with distinct large hilum, more than half its length ............ 2

b. Nutlets with small hilum, less than fourth its length ................. 6

2a. Nutlets obovoid or narrowly obovoid, length $2.3-3 \mathrm{~mm} \ldots \ldots \ldots \ldots . \ldots 3$

b. Nutlets ellipsoid or nearly ellipsoid, length $1.2-2.2 \mathrm{~mm} \ldots \ldots \ldots \ldots \ldots .4$

3a.Nutlets $0.6-0.8 \mathrm{~mm}$ wide; sculpture reticulate above (1/3 L.), transversely rugose in remaining its length. $(2 / 3 \mathrm{~L}$.); anticlinal boundaries thin

Ajuga chamaepitys subsp. tridactylites

b. Nutlets 1.3-1.6 mm wide; sculpture reticulate; anticlinal boundaries very thick Ajuga iva

4a. Hilum 0.8-0.82x0.77-0.81 mm; nutlets $1.2-1.5 \times 0.7-1 \mathrm{~mm}$; dark brown to black

Teucrium leucocladum

b. Hilum1-1.2x0.98-1.1 mm; nutlets 1.6-2.2x1-1.4 mm; dark brown ..... 5

5a. Nutlets ellipsoid-obovoid; periclinal cell wall sculpture striatefavulariate; anticlinal boundaries slightly raised ... Teucrium decaisnei

b. Nutlets ellipsoid-oblong; periclinal cell wall cell sculpture striate-to microcrimped papilla; anticlinal boundaries raised ... Teucrium polium

6a. Nutlets sculpture distinct reticulate or nearly reticulate $\ldots \ldots \ldots \ldots \ldots \ldots . .7$

b. Nutlets sculpture not reticulate ................................ 24

7a. Anticlinal boundaries channelled ............................... 8

b. Anticlinal boundaries raised or slightly raised ................... 12

8a. Nutlets $0.8-1 \times 0.4-0.6 \mathrm{~mm}$; with base obtuse; hilum 0.05-0.1 mm wide . 9

b. Nutlets 1.8-2.8x1-2.4 mm; with base rounded or shortly attenuate-

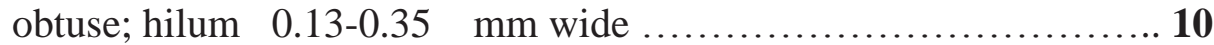

9a.Sculpture of periclinal cell wall circular striate; anticlinal boundaries straight; hilum ovoid, $0.06-0.1 \mathrm{~mm}$ long Micromeria nervosa

b. Sculpture of periclinal cell wall striate to micro-reticulate; anticlinal boundaries undulate; hilum obovoid, 0.12-0.15 mm long 
10a.Nutlets oblong, with glandular hairs at apex ......... Marrubium alysson b. Nutlets obovoid or ellipsoid- obovoid, not hairy at apex ............ 11

11a. Nutlet dark brown to black; hilum $0.3-0.35 \times 0.35-0.4 \mathrm{~mm}$; sculpture of periclinal wall striate-rugose Stachys aegyptiaca

b. Nutlet brown; hilum $0.15-0.18 \times 0.13-0.15 \mathrm{~mm}$; sculpture of periclinal wall striate-to micro-reticulate Thymus bovei

12a. Nutlet surface with darker net veins; sculpture reticulte-foveate ..... 13

b. Nutlet surface without darker net veins; sculpture not as above ..... 14

13a. Nutlets 1.8-2.2mm long; semi-matte; anticlinal boundaries raised, thin, straite Salvia multicaulis

b. Nutlets 1.2-1.5mm long; glossy; anticlinal boundaries slightly raised, thick, not straite (smooth) Salvia spinosa

14a. Nutlet apex truncate; ventral surface with distinct mid rib ......... 15

b. Nutlet apex rounded ; ventral surface with mostly distinct mid rib toward base 16

15a.Nutlet dark brown with irregular whitish spots; base truncate with an elongated light coloured elaiosome cells ; sculpture of periclinal cell wall ruminate to micro-reticulate, concave ...... Lamium amplexicaule

b. Nutlets brown; shortly attenuate towards base, sculpture of periclinal cell wall striate-foveate, concave with sunken center

Otostegia fruticosa

16a. Hilum bilobed or broad obcordate-rounded, 0.3-0.6 mm wide .... 17

b. Hilum not bilobed, $0.05-0.28 \mathrm{~mm}$ wide ....................... 20

17a. Nutlets with glandular hairs; dorsal surface convex-trigonous ...... 18

b. Nutlets glabrous; dorsal surface convex ....................... 19 18a. Nutlets 1.2-1.4x0.6-0.7mm; anticlinal boundaries thick; periclinal cell wall slightly concave with sunken center Lavandula coronopifolia

b. Nutlets $1.5-1.8 \times 0.8-1 \mathrm{~mm}$; anticlinal boundaries thin; periclinal cell wall flat- slightly concave Lavandula pubescens

19a. Nutlets oblong-ellpisoid or obovoid, 1.6-2x0.9-1.1mm; sculpture reticulate-tuberculate; anticlinal boundaries thin Nepta septemcrenata

b. Nutlets subglobose, $0.6-0.7 \times 0.4-0.5 \mathrm{~mm}$; sculpture reticulate; anticlinal boundaries thick ...... Mentha longifolia subsp. Typhoides

20a. Nutlets brown or dark brown; venteral surface hairy at apex ..... 21

b. Nutlets black; venteral surface glabrous ....................... 22

21a. Nutlet sculpture reticulate; sculpture of periclinal cell wall striate-to micro-reticulate; anticlinal boundaries thick Ballota undulata 
b. Nutlets sculpture reticulate-verrucate ; sculpture of periclinal wall striate-favulariate; anticlinal boundaries thin ...... Marrubium vulgare

22a. Nutlets 1.2-1.4mm long; sculpture reticulate -verrucate; anticlinal boundaries \pm thick; sculpture of periclinal cell wall favulariate

Salvia lanigera

b. Nutlets 1.8-2.2 mm long; sculpture reticulate; anticlinal boundaries thin; sculpture of periclinal cell wall striate 23

23a. Nutlets semi-matte, oblong or oblong-ellipsoid, 0.8-0.9 mm wide; hilum obovoid Salvia aegyptiaca

b. Nutlets matte, oblanceoloid-ellipsoid, 1.1-1.2 mm wide; hilum ellipsoid .................................................... Salvia deserti

24a. Nutlets $0.7-1 \times 0.2-0.7 \mathrm{~mm}$; yellowish or orange brown ............. 25

b. Nutlets $2.5-5.5 \times 2-3 \mathrm{~mm}$; pale to dark brown ....................... 26

25a. Nutlets narrow oblong, sculpture longitudinal coase rugose Micromeria sinaica

b. Nutlets subglobose to broadly ovoid, sculpture ruminate Origanum syriacum

26a. Nutlets obovoid- trigonous; apex truncate with small beak; surface of nutlets without vines Phlomis aurea

b. Nutlets globose, subglobose-ellipsoid, apex rounded; surface of nutlets with darker net viens ....................................... 27

27a. Sculpture smooth-slightly tuberculate; nutlets $3.2-3.7 \mathrm{~mm}$ long Salvia palaestina

b. Sculpture sclariform-rugose; nutlets 2.4-2.6mm long ... Salvia sclarea

\section{References}

Barthlott W. 1981. Epidermal and seed surface characters of plants: systematic applicability and some evolutionary aspects. Nord. J. Bot. 1: 345-355.

Bendiksby, M., Thorbek, L., Scheen, A., Lindqvist, C. \& Ryding, O. 2011. An updated phylogeny and classification of Lamiaceae subfamily Lamioideae. Taxon. 60: 471- 484.

Boulos, L. 2002. Flora of Egypt: Volume III (Verbenaceae - Compositae). Al-Hadara Publishing, Cairo, Egypt.

Boulos, L. 2009. Flora of Egypt checklist, Revised Annotated Edition. AlHadara Publishing, Cairo, Egypt. 
Cantino, P. D. 1990. The phylogenetic significance of stomata and trichomes in the Labiatae and Verbenaceae. J. Arnold. Arboretum. 71: 323-370.

Cantino, P. D. 1992. Evidence for the polyphyletic origin of the Labiatae. Ann. Missouri. Bot. Gard. 79: 361-379

Demissew, S.\& Harley, M. M. 1992.Trichome, seed surface and pollen characters in Stachys (Labiatae) in Tropical Africa. In:Harley R. M., Reynolds T. (eds), Advances in Labiatae science. Kew: Roy. Bot. Gard.149-166.

El-Hadidi, M. N. \& Fayed, A. A. 1995. Materials for Excursion Flora of Egypt. Cairo University Herbarium, Täeckholmia. 15: 1-223.

El-Husseini, N. M. 1986. Flora of Egypt. Taxonomic revision of Labiatae. Unpublisded Ph.D. Thesis, Cairo University, Egypt.

Guerin, G.R . 2005. Nutlet morphology in Hemigenia R.Br. and Microcorys R.Br. (Lamiaceae). Pl. Syst. Evol. 254: 49-68.

Harley, R. M., Atkins, S., Budantsev, A. L., Cantino, P. D., Conn, B. J., Grayer, R.,Harley, M.M.,De Kok, R.,Krestovskaja, T.,Morales, R.,Paton,A. J., Ryding, O.\& Upson, T. 2004. Labiatae. In: Kubitzki, $\mathrm{K}$ (ed.), the families and genera of vascular plants 7, Flowering plants. Dicotyledons: Lamiales, 167-275. Springer, Stuttgart.

Hassan, T. \& Dar, G.H. 2012. Nutlet Micromorphology In Selected Species of Nepeta L. (Lamiaceae) in Kashmir Himalaya. Iran. J. Bot. 18(2): 262-269.

Hedge, I. C. 1992. A global survey of the biogeography of the Labiatae. In: Harley, R. M., Reynolds T. (eds.), Advances in Labiatae science. Kew, Roy. Bot. Gard., 7-17.

Heywood, V. H. 1971. Scanning electron microscopy. Systematic and evolutionary applications, London.

Husain, S. Z., Marin, P. D., Siliĉ, C., Qaiser, M.\& Petkoviĉ, B. (1990). A micromorphological study of some representative genera in the tribe Saturejeae (Lamiaceae). Bot. J. Linn. Soc. 103: 59-80.

Karhraman, A. \& Dògan, M. 2010. Comparative study of Salvia limbata C. A. and S. palaestina Bentham (sect. Aethiopis Bentham, Labiatae) from East Anatolia, Turkey. Acta Bot. Croat. 69(1): 47- 64.

Marin, P. D., Petkoviĉ, B. P. \& Duletiĉ, S. 1994. Nutlet sculpturing of selected Teucrium species (Lamiaceae): a character of taxonomic significance. Pl. Syst. Evol. 192: 199-214. 
Marin, P. D., Duletiĉ, S \& Petkoviĉ, B. P. 1996. Nutlet ornamentation in selected Salvia L. species (Lamiaceae). Fl. Medit. 6: 203 - 211.

Mousavi, S. M., Jafari, A. \& Najafi,, S. 2013. Nutlet Micromorphological Study on Salvia L. (Lamiaceae) from NE Iran," AJPS, 4: 1457-1460.

Özkan, M., Aktas, K., Özdemir, C., \& Guerin, G. 2009. Nutlet morphology and its taxonomic utility in Salvia (Lamiaceae : Mentheae) from Turkey. Acta Bot. Croat. 68(1): 105- 115.

Oran, S. A. 1996. Ultrastructure of nutlet surface of the genus Salvia L. in Jordan and the neighbouring countries. Dirasat, Natural and Engineering Sciences.23: 393-408.

Packham, J. R. 1983. Biological flora of the British Isles. Lamiastrum galeobdolon (L.) Ehrend. \& Polatschek (Galeobdolon luteum Hudson; Lamium galeobdolon (L.) Nath.). J. Ecol. 71: 975-997.

Ryding, O. 1993. Pericarp structure of Leucas and related genera (Lamiaceae subfam. Lamioideae). Nord. J. Bot. 13 (6): 637- 646.

Ryding, O. 1994. Pericarp structure and phylogeny of Lamiaceae subfamily Pogostemonoideae. Nord. J. Bot. 14: 59-63.

Ryding, O. 1998. Phylogeny of the Leucas Group (Lamiaceae). Sys. Bot. 23(2): 235-237.

Salmaki, Y., Zarre, S.\& Jamzad, Z. 2008. Nutlet micromorphology and its systematic implication in Stachys L. (Lamiaceae) In Iran. Feddes Repert. 119: 607-621.

Stace, C. A. 1989. Plant taxonomy and biosystematics, 2nd ed. Cambridge University Press, Cambridge.

Stearn, W. T. 1983. Botanical Latin. 3rd (ed.) rev., David \& Charles, London.

Strasburger, E. 1978. Lehrbuch der Botanik für Hochschulen 31 ed., (neubearbeitet von D.V. Denffer, F.Ehrendorfer, K. Mägdefrau, H. Ziegler) Gustav Fischer Verlag. Stuttgart, New York.

Stuessy, T. F. 1990. Plant taxonomy, the systematic evaluation of comparative data. Columbia University Press, New York.

Täckholm, V. 1974. Students' Flora of Egypt (ed.2). Cairo University, Egypt.

Tarimcilar, G., Yilmaz, Ö, Daskin, R. \& Kaynak, G. 2013. Nutlet morphology and its taxonomic significance in the genus Mentha L. (Lamiaceae) from Turkey. Bangl. J. Plant Taxon. 20 (1): 9 - 18. 
Wojciechowska, B. 1972. Morphology and anatomy of fruits of Scutellaria Chaiturus, Galeobdolon, and Sideritis of family Labiatae. Monogr. Bot. 37: 137-168 (in Polish). 
Table 1. Nutlet specimens examined; their sub familial classification (according to Harley 2004) and collection details.

\begin{tabular}{|c|c|}
\hline Taxa & Collection Data \\
\hline $\begin{array}{l}\text { Subfamily: Ajugoideae kostel. (1843) } \\
\text { 1- Ajuga chamaepitys (L.) Schreb. } \\
\text { subsp. tridactylites (Benth.) P.H.Davis }\end{array}$ & $\begin{array}{l}\text { S. Sinai, E' slopes of Jebel Katherine, } 1900 \text { - } \\
2250 \mathrm{~m}, 33^{\circ} 58^{\prime} \text { E, } 28^{\circ} 31^{\prime} \text { N, 28.4.1991, E. Gamal } \\
\text { Eldin, s.n.SCU-I. }\end{array}$ \\
\hline 2- Ajuga iva (L.) Schreb. & $\begin{array}{l}\text { Mersa- Matruh, the road of Sallum, 16.4.2010, } \\
\text { A.El Banhawy, s.n.SCU-I. }\end{array}$ \\
\hline 3- Teucrium decaisnei C.Presl & $\begin{array}{l}\text { S. Sinai, Wadi El-Deir, 31.8.2001, W. Kamel, } \\
\text { s.n.SCU-I. }\end{array}$ \\
\hline 4- Teucrium leucocladum Boiss. & $\begin{array}{l}\text { S. Sinai, Wadi Teffaha, } 33^{\circ} 55^{\prime} \text { E, } 28^{\circ} 34^{\prime} \text { N, } \\
\text { 30.8.2001, W. Kamel, s.n.SCU-I. }\end{array}$ \\
\hline 5- Teucrium polium $\mathrm{L}$. & $\begin{array}{l}\text { S. Sinai, Wadi Gebal, 13.7.2002, M. Soliman, } \\
\text { s.n.SCU-I. }\end{array}$ \\
\hline Subfamily: Lamioideae Harley (2003) & \\
\hline 6-Ballota undulata (Fresen.) Benth. & $\begin{array}{l}\text { S. Sinai, Kahf El-Ghola, 21.6.1999, W. Kamel, } \\
\text { s.n.SCU-I. }\end{array}$ \\
\hline 7-Lamium amplexicaule L. & $\begin{array}{l}\text { S. Sinai, Wadi El-Arbaeen, El-Deir garden,33 } \\
57^{\prime} \text { E, } 28^{\circ} 33^{\prime} \text { N, 3.5.2004, W. Kamel, s.n.SCU-I. }\end{array}$ \\
\hline 8-Marrubium alysson $\mathrm{L}$. & $\begin{array}{l}\text { Alexanderia - Matruh road Kilo 45, 31.8.1984, } \\
\text { W. Kamel s.n.SCU-I. }\end{array}$ \\
\hline 9-Marrubium vulgare $\mathrm{L}$. & $\begin{array}{l}\text { Sallum road , toward W. Mersa- Matruh, } \\
\text { 15.4.2010, A.El Banhawy, s.n.SCU-I. }\end{array}$ \\
\hline 10-Otostegia fruticosa (Forssk.) Penz. & $\begin{array}{l}\text { S. Sinai, Wadi Isla , 1.10.1990, E.Gamal Eldin, } \\
\text { s.n.SCU-I. }\end{array}$ \\
\hline 11-Phlomis aurea Decne. & $\begin{array}{l}\text { S. Sinai, Wadi El-Arbaeen, } 33^{\circ} 57^{\prime} \text { E, } 28^{\circ} 32^{\prime} \text { N, } \\
\text { 2.11.2009, W. Kamel, s.n.SCU-I. }\end{array}$ \\
\hline 12- Stachys aegyptiaca Pers. & $\begin{array}{l}\text { S. Sinai, Wadi El-Arbaeen, 5.11.2009, W. Kamel, } \\
\text { s.n.SCU-I. }\end{array}$ \\
\hline \multicolumn{2}{|c|}{ Subfamily: Nepetoideae (Dumort.) Luerss.( 1882) } \\
\hline 13-Lavandula coronopifolia Poir. & $\begin{array}{l}\text { S. Sinai, Wadi Gharba, 2.6.2001, M. Zaghoul, } \\
\text { s.n.SCU-I. }\end{array}$ \\
\hline 14-Lavandula pubescens Decne. & $\begin{array}{l}\text { S. Sinai, Wadi Tala'a , 24.4.2009, W. Kamel, } \\
\text { s.n.SCU-I. }\end{array}$ \\
\hline $\begin{array}{l}\text { 15-Mentha longifolia (L.) Huds.subsp. } \\
\text { typhoides (Briq) Harley }\end{array}$ & $\begin{array}{l}\text { S. Sinai, Wadi Isla , 1.10.1990, E.Gamal Eldin, } \\
\text { s.n.SCU-I. }\end{array}$ \\
\hline 16-Micromeria nervosa (Desf.) Benth. & $\begin{array}{l}\text { N. Sinai, Ain El-Gedeirat , 3.5.1988, W. Kamel, } \\
\text { s.n.SCU-I. }\end{array}$ \\
\hline 17-Micromeria sinaica Benth. & $\begin{array}{l}\text { S. Sinai, Wadi El-Deir, 30.5.1991, E.Gamal } \\
\text { Eldin, s.n.SCU-I. }\end{array}$ \\
\hline 18-Nepeta septemcrenata Benth. & $\begin{array}{l}\text { S. Sinai, Kahf El-Ghola, 3.12.1993, W. Kamel, } \\
\text { s.n.SCU-I. }\end{array}$ \\
\hline
\end{tabular}


Table 1 (Continued)

\begin{tabular}{|c|c|}
\hline $\begin{array}{l}\text { 19-Origanum syriacum L. subsp. } \\
\text { sinaicum (Boiss.) Greuter\&Burdet }\end{array}$ & $\begin{array}{l}\text { S. Sinai, Wadi Abu Tuweita, 9.5.2009, A.El } \\
\text { Banhawy, s.n.SCU-I. }\end{array}$ \\
\hline 20-Salvia aegyptiaca $\mathrm{L}$. & $\begin{array}{l}\text { N. Sinai, before Nakhl by } 3 \mathrm{Km}, 3.4 .1996, \mathrm{~W} \text {. } \\
\text { Kamel, s.n.SCU-I. }\end{array}$ \\
\hline 21-Salvia deserti Decne. & $\begin{array}{l}\text { S. Sinai, Wadi El- Rahaa, } 1480-1500 \text { m, } 33^{\circ} 56^{\prime} \\
\text { E, } 28^{\circ} 35^{\prime} \text { N, 23.4.12009 W. Kamel, s.n.SCU-I. }\end{array}$ \\
\hline 22-Salvia lanigera Poir. & $\begin{array}{l}\text { N. Sinai, El-Qusaima, H.Soliman garden, } \\
\text { 2.4.2009. W. Kamel, s.n.SCU-I. }\end{array}$ \\
\hline 23-Salvia multicaulis Vahl & $\begin{array}{l}\text { S. Sinai, Wadi Abu Tuweita, 9.5.2009, A.El } \\
\text { Banhawy, s.n.SCU-I. }\end{array}$ \\
\hline 24-Salvia palaestina Benth. & $\begin{array}{l}\text { S. Sinai, Wadi Tobouq, } 1775 \mathrm{~m}, 33^{\circ} 56^{\prime} \mathrm{E}, \\
28^{\circ} 32^{\prime} \text { N, 8.5.2009, A.El Banhawy, s.n.SCU-I. }\end{array}$ \\
\hline 25-Salvia sclarea $\mathrm{L}$. & $\begin{array}{l}\text { S. Sinai, Wadi Gebal, 10.5.2009, A.El Banhawy, } \\
\text { s.n.SCU-I. }\end{array}$ \\
\hline 26-Salvia spinosa $\mathrm{L}$. & $\begin{array}{l}\text { S. Sinai, Scientific station in St. Katherine city, } \\
33^{\circ} 57^{\prime} \text { E, } 28^{\circ} 33^{\prime} \text { N, 3.5.2004, W. Kamel, } \\
\text { s.n.SCU-I. }\end{array}$ \\
\hline 27-Thymus bovei Benth. & $\begin{array}{l}\text { S. Sinai, Wadi Gebal, 3.12.2011, M. Soliman, } \\
\text { s.n.SCU-I. }\end{array}$ \\
\hline 28-Thymus decussatus Benth. & $\begin{array}{l}\text { S. Sinai, Wadi El Fara'a, 4.5.2004, W. Kamel, } \\
\text { s.n.SCU-I. }\end{array}$ \\
\hline
\end{tabular}


Table 2. Nutlet macromorpholog of studied taxa.

\begin{tabular}{|c|c|c|c|c|c|c|c|c|c|}
\hline \multirow[b]{2}{*}{ Taxa } & \multicolumn{9}{|c|}{ Characters } \\
\hline & Shape & $\begin{array}{c}\mathbf{L} \mathbf{X ~ W} \\
(\mathbf{m m})\end{array}$ & Colour & Luster & $\begin{array}{l}\text { Dorsal } \\
\text { surface }\end{array}$ & $\begin{array}{l}\text { Ventral } \\
\text { midrib }\end{array}$ & Apex & Base & $\begin{array}{c}\text { Presence of } \\
\text { glands }\end{array}$ \\
\hline $\begin{array}{l}\text { Subfamily: Ajugoideae } \\
\text { 1- Ajuga chamaepitys (L.) } \\
\text { Schreb. subsp. tridactylites } \\
\text { (Benth.) P.H.Davis }\end{array}$ & Narrowly obovoid & $\begin{array}{c}2.3-2.8 X \\
0.6-0.8\end{array}$ & $\begin{array}{l}\text { Brown or } \\
\text { dark brown }\end{array}$ & Matte & Convex & Indistinct & Rounded & Truncate & - \\
\hline 2- Ajuga iva (L.) Schreb. & Obovoid & $\begin{array}{l}2.5-3 \mathrm{X} \\
1.3-1.6 \\
\end{array}$ & $\begin{array}{l}\text { Black or } \\
\text { greyish }\end{array}$ & Matte & Convex & Indistinct & Rounded & Rounded & - \\
\hline 3- Teucrium decaisnei C.Presl & Ellipsoid -obovoid & $\begin{array}{l}1.6-2 \times 1- \\
1.3\end{array}$ & Dark brown & Matte & Convex & Indistinct & Rounded & Rounded & - \\
\hline 4- Teucrium leucocladum Boiss. & Ellipsoid & $\begin{array}{c}1.2-1.5 X \\
0.7-1\end{array}$ & $\begin{array}{l}\text { Dark brown } \\
\text { to black }\end{array}$ & Matte & Convex & Indistinct & Rounded & Rounded & - \\
\hline 5- Teucrium polium $\mathrm{L}$. & Ellipsoid -oblong & $\begin{array}{c}1.8-2.2 \mathrm{X} \\
1.1-1.4 \\
\end{array}$ & Dark brown & Matte & Convex & Indistinct & Rounded & Rounded & - \\
\hline $\begin{array}{l}\text { Subfamily: Lamioideae } \\
\text { 6-Ballota undulata (Fresen.) } \\
\text { Benth. }\end{array}$ & Obovoid & $\begin{array}{l}1.8-2.2 \mathrm{X} \\
1-1.3\end{array}$ & Dark brown & Matte & Convex & \pm Distinct & Rounded & Obtuse & \pm \\
\hline 7-Lamium amplexicaule L. & $\begin{array}{l}\text { Prolonged } \\
\text { obovoid }\end{array}$ & $\begin{array}{l}2-2.4 X \\
1.2-1.3\end{array}$ & $\begin{array}{l}\text { Dark brown } \\
\text { with irregular } \\
\text { whitish spots }\end{array}$ & Matte & Convex & Distinct & $\begin{array}{l}\text { Oblique } \\
\text { truncate }\end{array}$ & $\begin{array}{l}\text { Truncate with } \\
\text { elongated cells base }\end{array}$ & - \\
\hline 8-Marrubium alysson L. & Oblong & $\begin{array}{c}1.8-2 \mathrm{X} \\
1-1.2\end{array}$ & Dark brown & Matte & Convex & $\begin{array}{c}\text { Distinct } \\
\text { toward base }\end{array}$ & Rounded & Rounded & + at apex \\
\hline 9-Marrubium vulgare L. & obovoid- ellipsoid & $\begin{array}{l}2-2.2 \mathrm{X} \\
1.1-1.3\end{array}$ & Brown & $\begin{array}{l}\text { Semi- } \\
\text { matte }\end{array}$ & Convex & $\begin{array}{l}\text { Distinct } \\
\text { toward base }\end{array}$ & Rounded & $\begin{array}{l}\text { Rounded } \pm \text { pointed } \\
\text { tip }\end{array}$ & $\begin{array}{c}+ \text { at apex of } \\
\text { ventral surface }\end{array}$ \\
\hline $\begin{array}{l}\text { 10-Otostegia fruticosa (Forssk.) } \\
\text { Penz. }\end{array}$ & $\begin{array}{l}\text { Widely obovoid- } \\
\text { trigonous }\end{array}$ & $\begin{array}{l}1.3-1.5 \mathrm{X} \\
1.1-1.3\end{array}$ & Brown & Matte & \pm Convex & Distinct & Truncate & Shortly attenuate & 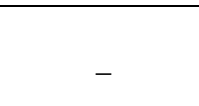 \\
\hline 11-Phlomis aurea Decne. & $\begin{array}{l}\text { Obovoid- } \\
\text { trigonous }\end{array}$ & $\begin{array}{l}4.3-5.5 X \\
1.9-2.5\end{array}$ & $\begin{array}{l}\text { Brown to dark } \\
\text { brown }\end{array}$ & Matte & Convex & Distinct & $\begin{array}{l}\text { Truncate with } \\
\text { small beak }\end{array}$ & $\begin{array}{l}\text { Very shortly } \\
\text { attenuate - obtuse }\end{array}$ & - \\
\hline 12- Stachys aegyptiaca Pers. & Obovoid & $\begin{array}{c}2.2-2.5 \mathrm{X} \\
1.2-1.4 \\
\end{array}$ & $\begin{array}{c}\text { Dark brown } \\
\text { to black }\end{array}$ & Matte & Convex & $\begin{array}{c}\text { Distinct } \\
\text { toward base }\end{array}$ & $\begin{array}{c}\text { Rounded or } \pm \\
\text { mucronate }\end{array}$ & Rounded & - \\
\hline $\begin{array}{l}\text { Subfamily: Nepetoideae } \\
\text { 13-Lavandula coronopifolia Poir. }\end{array}$ & Oblong & $\begin{array}{c}1.2-1.4 \mathrm{X} \\
0.6-0.7\end{array}$ & Brown & $\begin{array}{l}\text { Semi- } \\
\text { matte }\end{array}$ & $\begin{array}{l}\text { Convex- } \\
\text { trigonous }\end{array}$ & Indistinct & Rounded & Rounded & + \\
\hline 14-Lavandula pubescens Decne. & $\begin{array}{l}\text { Oblong or oblong- } \\
\text { ellipsoid }\end{array}$ & $\begin{array}{l}1.5-1.8 \mathrm{X} \\
0.8-1\end{array}$ & $\begin{array}{l}\text { Pale brown or } \\
\text { brown }\end{array}$ & Matte & $\begin{array}{l}\text { Convex- } \\
\text { trigonous }\end{array}$ & Indistinct & Rounded & Rounded & + \\
\hline
\end{tabular}


Table 2 (Continued)

\begin{tabular}{|c|c|c|c|c|c|c|c|c|c|}
\hline $\begin{array}{l}\text { 15-Mentha longifolia (L.). } \\
\text { Huds.subsp typhoides(Briq.)Harley }\end{array}$ & Subglobose & $\begin{array}{c}0.6-0.7 \mathrm{X} \\
0.4-0.5\end{array}$ & $\begin{array}{l}\text { Brown to dark } \\
\text { brown }\end{array}$ & $\begin{array}{l}\text { Semi- } \\
\text { matte }\end{array}$ & Convex & $\begin{array}{c}\text { Distinct } \\
\text { toward base }\end{array}$ & Rounded & Obtuse & - \\
\hline $\begin{array}{l}\text { 16-Micromeria nervosa (Desf.) } \\
\text { Benth. }\end{array}$ & Oblong & $\begin{array}{c}0.8-0.9 \mathrm{X} \\
0.4-0.5\end{array}$ & Brown & $\begin{array}{l}\text { Semi- } \\
\text { matte }\end{array}$ & \pm Convex & Indistinct & Rounded & Obtuse & - \\
\hline 17-Micromeria sinaica Benth. & $\begin{array}{c}\text { Narrowly } \\
\text { oblong }\end{array}$ & $\begin{array}{l}0.7-1 \mathrm{X} \\
0.2-0.3 \\
\end{array}$ & $\begin{array}{c}\text { Yellowish } \\
\text { brown }\end{array}$ & $\begin{array}{l}\text { Semi- } \\
\text { matte }\end{array}$ & Convex & Indistinct & Obtuse & Obtuse & - \\
\hline 18-Nepeta septemcrenata Benth. & $\begin{array}{l}\text { Oblong - } \\
\text { ellipsoid or } \\
\text { obovoid }\end{array}$ & $\begin{array}{l}1.6-2 \mathrm{X} \\
0.9-1.1\end{array}$ & $\begin{array}{l}\text { Dark brown or } \\
\text { black }\end{array}$ & Matte & Convex & Indistinct & Rounded & Rounded & - \\
\hline $\begin{array}{l}\text { 19-Origanum syriacum L. subsp. } \\
\text { sinaicum (Boiss.) Greuter\&Burdet }\end{array}$ & $\begin{array}{l}\text { Subglobose to } \\
\text { broadly ovoid }\end{array}$ & $\begin{array}{c}0.7-0.9 X \\
0.5-0.7\end{array}$ & Orange brown & $\begin{array}{l}\text { Semi- } \\
\text { matte }\end{array}$ & Convex & Indistinct & Rounded & Obtuse & - \\
\hline 20-Salvia aegyptiaca L. & $\begin{array}{c}\text { Oblong or } \\
\text { oblong- } \\
\text { Ellipsoid }\end{array}$ & $\begin{array}{l}1.8-2 X \\
0.8-0.9\end{array}$ & black & $\begin{array}{l}\text { Semi- } \\
\text { matte }\end{array}$ & Convex & Indistinct & Rounded & $\begin{array}{l}\text { shortly attenuate - } \\
\text { obtuse }\end{array}$ & - \\
\hline 21-Salvia deserti Decne. & $\begin{array}{c}\text { Oblanceoloid- } \\
\text { ellipsoid }\end{array}$ & $\begin{array}{c}1.8-2.2 \mathrm{X} \\
1.1-1.2 \\
\end{array}$ & black & Matte & Convex & Indistinct & Rounded & $\begin{array}{c}\text { shortly attenuate - } \\
\text { obtuse }\end{array}$ & - \\
\hline 22-Salvia lanigera Poir. & $\begin{array}{c}\text { Obovoid- } \\
\text { ellipsoid }\end{array}$ & $\begin{array}{c}1.2-1.4 \mathrm{X} \\
0.6-0.7\end{array}$ & black & $\begin{array}{l}\text { Semi- } \\
\text { matte }\end{array}$ & Convex & Indistinct & Rounded & $\begin{array}{c}\text { shortly attenuate - } \\
\text { obtuse }\end{array}$ & - \\
\hline 23-Salvia multicaulis Vahl & $\begin{array}{l}\text { Subglobose- } \\
\text { obovoid }\end{array}$ & $\begin{array}{l}1.8-2.2 \mathrm{X} \\
1.2-1.4\end{array}$ & $\begin{array}{c}\text { dark brown with } \\
\text { fine longitudinal } \\
\text { net veins }\end{array}$ & $\begin{array}{l}\text { Semi- } \\
\text { matte }\end{array}$ & Convex & Indistinct & Rounded & Rounded & - \\
\hline 24-Salvia palaestina Benth. & $\begin{array}{c}\text { Globose- } \\
\text { subglobose }\end{array}$ & $\begin{array}{c}3.2-3.7 \mathrm{X} \\
2.5-3 \\
\end{array}$ & $\begin{array}{l}\text { Pale brown with } \\
\text { darker net veins }\end{array}$ & Glossy & Convex & Indistinct & Rounded & Obtuse & - \\
\hline 25-Salvia sclarea L. & $\begin{array}{l}\text { Subglobose- } \\
\text { ellipsoid }\end{array}$ & $\begin{array}{c}2.4-2.6 \mathrm{X} \\
2-2.4\end{array}$ & $\begin{array}{c}\text { Brown with } \\
\text { darker net veins }\end{array}$ & $\begin{array}{l}\text { Semi- } \\
\text { matte }\end{array}$ & Convex & Indistinct & Rounded & Truncate & - \\
\hline 26-Salvia spinosa $\mathrm{L}$. & $\begin{array}{c}\text { Subglobose- } \\
\text { globose }\end{array}$ & $\begin{array}{c}1.2-1.5 \mathrm{X} \\
1-1.4 \\
\end{array}$ & $\begin{array}{c}\text { Brown with } \\
\text { darker net veins }\end{array}$ & Glossy & Convex & Indistinct & Rounded & Obtuse & - \\
\hline 27-Thymus bovei Benth. & $\begin{array}{l}\text { Ellipsoid- } \\
\text { obovoid or } \\
\text { obovoid }\end{array}$ & $\begin{array}{l}2.5-2.8 X \\
2.2-2.4\end{array}$ & Brown & $\begin{array}{l}\text { Semi- } \\
\text { matte }\end{array}$ & Convex & Indistinct & Rounded & $\begin{array}{l}\text { shortly attenuate - } \\
\text { obtuse }\end{array}$ & - \\
\hline 28-Thymus decussatus Benth. & Ovoid- oblong & $\begin{array}{l}0.9-1 X \\
0.5-0.6 \\
\end{array}$ & Brown & $\begin{array}{l}\text { Semi- } \\
\text { matte }\end{array}$ & Convex & Indistinct & Rounded & $\begin{array}{c}\text { Obtuse with } \pm \text { small } \\
\text { beak }\end{array}$ & - \\
\hline
\end{tabular}


Table 3. Nutlet micromorphology of studied taxa.

\begin{tabular}{|c|c|c|c|c|c|c|c|c|c|}
\hline \multirow[b]{2}{*}{ Taxa } & \multicolumn{3}{|c|}{ Testa appearance } & \multicolumn{4}{|c|}{ Anticlinal wall } & \multicolumn{2}{|c|}{ Periclinal wall } \\
\hline & $\begin{array}{l}\text { Multicellular } \\
\text { Sculpture }\end{array}$ & $\begin{array}{c}\text { Homogeneity } \\
\text { of cell size }\end{array}$ & $\begin{array}{c}\text { Shape of } \\
\text { epidermal } \\
\text { cell }\end{array}$ & Level & Undulation & Thickness & Sculpture & Level & Sculpture \\
\hline $\begin{array}{l}\text { Subfamily: Ajugoideae } \\
\text { 1- Ajuga chamaepitys (L.) Schreb. } \\
\text { subsp. tridactylites (Benth.) P.H.Davis }\end{array}$ & $\begin{array}{l}\text { Upper portion } \\
\text { (1/3L.) reticulate, } \\
\text { transversely rugose } \\
\text { (2/3L.) }\end{array}$ & Irregular & $\begin{array}{l}\text { Penta-, } \\
\text { hexagonal }\end{array}$ & Raised & \pm Straight & Thin & Striate & Concave & $\begin{array}{c}\text { Striate- } \\
\text { favulariate }\end{array}$ \\
\hline 2- Ajuga iva (L.) Schreb. & Reticulate & Irregular & $\begin{array}{l}\text { Penta-, } \\
\text { hexagonal } \\
\text { elongated, } \\
\text { rounded }\end{array}$ & Raised & Undulate & Very thick & Undulate & Concave & Smooth \\
\hline 3- Teucrium decaisnei C.Presl & $\begin{array}{l}\text { Reticulate or } \\
\text { reticulate- } \\
\text { colliculate } \\
\end{array}$ & Regular & $\begin{array}{c}\text { Penta-, } \\
\text { hexagonal }\end{array}$ & $\begin{array}{l}\text { Slightly } \\
\text { raised }\end{array}$ & Straight & Thin & Striate & $\begin{array}{l}\text { Slightly } \\
\text { concave }\end{array}$ & $\begin{array}{l}\text { Striate - } \\
\text { favulariate }\end{array}$ \\
\hline 4- Teucrium leucocladum Boiss. & $\begin{array}{l}\text { Reticulate or } \\
\text { reticulate- } \\
\text { colliculate } \\
\end{array}$ & Regular & $\begin{array}{l}\text { Rounded, } \\
\text { Pentagonal }\end{array}$ & $\begin{array}{l}\text { Slightly } \\
\text { raised }\end{array}$ & Straight & Thin & Striate & Concave & Favulariate \\
\hline 5- Teucrium polium $\mathrm{L}$. & $\begin{array}{l}\text { Reticulate or } \\
\text { reticulate- } \\
\text { colliculate } \\
\end{array}$ & Irregular & $\begin{array}{l}\text { Penta-, } \\
\text { hexagonal }\end{array}$ & Raised & Straight & Thin & Striate & Concave & $\begin{array}{l}\text { Striate to micro- } \\
\text { crimped papilla }\end{array}$ \\
\hline $\begin{array}{l}\text { Subfamily: Lamioideae } \\
\text { 6-Ballota undulata (Fresen.) Benth. }\end{array}$ & Reticulate & Irregular & $\begin{array}{c}\text { Hexagonal } \\
\text { rounded }\end{array}$ & Raised & Straight & \pm Thick & Striate & Concave & $\begin{array}{l}\text { Striate to micro- } \\
\text { reticulate }\end{array}$ \\
\hline 7-Lamium amplexicaule L. & Reticulate & Irregular & $\begin{array}{c}\text { Tetra, penta-, } \\
\text { hexagonal }\end{array}$ & Raised & \pm Straight & Thin & Undulate & Concave & $\begin{array}{c}\text { Ruminate to } \\
\text { micro- reticulate }\end{array}$ \\
\hline 8-Marrubium alysson L. & $\begin{array}{l}\text { Reticulate- } \\
\text { verrucate }\end{array}$ & \pm Regular & $\begin{array}{c}\text { Penta-, } \\
\text { hexagonal }\end{array}$ & Channelled & Straight & Thin & Striate & $\begin{array}{l}\text { Flat- slightly } \\
\text { concave }\end{array}$ & $\begin{array}{c}\text { Smooth outer- } \\
\text { striate in center }\end{array}$ \\
\hline 9-Marrubium vulgare $\mathrm{L}$. & $\begin{array}{l}\text { Reticulate- } \\
\text { verrucate }\end{array}$ & Irregular & $\begin{array}{c}\text { Penta-, } \\
\text { hexagonal }\end{array}$ & Raised & Straight & Thin & Striate & Concave & $\begin{array}{c}\text { Striate- } \\
\text { favulariate }\end{array}$ \\
\hline 10-Otostegia fruticosa (Forssk.) Penz. & $\begin{array}{c}\text { Reticulate- } \\
\text { ruminate- }\end{array}$ & Regular & $\begin{array}{c}\text { Penta- } \\
\text { hexagonal }\end{array}$ & Raised & Straight & Thick & Striate & $\begin{array}{l}\text { Concave with } \\
\text { sunken center }\end{array}$ & Striate- foveate \\
\hline 11-Phlomis aurea Decne. & $\begin{array}{l}\text { Irregular rugose- } \\
\text { scrobiculate }\end{array}$ & Irregular & $\begin{array}{l}\text { Irregular, } \\
\text { elongated }\end{array}$ & \pm Raised & Straight & \pm Thick & Striate & Flat & Smooth \\
\hline
\end{tabular}


Table 3 (Continued )

\begin{tabular}{|c|c|c|c|c|c|c|c|c|c|}
\hline 12- Stachys aegyptiaca Pers. & Reticulate & Irregular & $\begin{array}{c}\text { Penta-, } \\
\text { hexagonal }\end{array}$ & Channelled & Straight & Thin & Striate & $\begin{array}{c}\text { Flat - slightly } \\
\text { concave }\end{array}$ & Striate- rugose \\
\hline $\begin{array}{l}\text { Subfamily: Nepetoideae } \\
\text { 13-Lavandula coronopifolia Poir. }\end{array}$ & Reticulate & Irregular & $\begin{array}{c}\text { Penta-, } \\
\text { hexagonal }\end{array}$ & Raised & Straight & Thick & Striate & $\begin{array}{l}\text { Concave with } \\
\text { sunken center }\end{array}$ & Striate \\
\hline 14-Lavandula pubescens Decne. & Reticulate & Irregular & $\begin{array}{c}\text { Penta, hexagonal, } \\
\text { elongated }\end{array}$ & $\begin{array}{c}\text { Slightly } \\
\text { Raised }\end{array}$ & Straight & Thin & Striate & $\begin{array}{c}\text { Flat - slightly } \\
\text { concave }\end{array}$ & Striate \\
\hline $\begin{array}{l}\text { 15- Mentha longifolia (L.) Huds } \\
\text { subsp typhoides(Briq.)Harley -. }\end{array}$ & Reticulate & Regular & $\begin{array}{c}\text { Penta-, } \\
\text { hexagonal, } \\
\text { rounded }\end{array}$ & $\begin{array}{l}\text { Slightly } \\
\text { raised }\end{array}$ & Straight & $\begin{array}{l}\text { Very } \\
\text { thick }\end{array}$ & Striate & Flat & $\begin{array}{l}\text { Striate with mostly } \\
\text { globular central } \\
\text { papilla }\end{array}$ \\
\hline $\begin{array}{l}\text { 16-Micromeria nervosa (Desf.) } \\
\text { Benth. }\end{array}$ & Reticulate & Regular & $\begin{array}{c}\text { Tetra-, penta-, } \\
\text { hexagonal, } \\
\text { elongated }\end{array}$ & Channelled & Straight & Thin & Striate & Flat & Circular striated \\
\hline 17-Micromeria sinaica Benth. & $\begin{array}{l}\text { Longitudinal } \\
\text { coarse rugose }\end{array}$ & Regular & Elongated & Raised & Straight & Thick & Ribbed & Concave & Smooth \\
\hline 18-Nepeta septemcrenata Benth. & $\begin{array}{l}\text { Reticulate- } \\
\text { tuberculate }\end{array}$ & Irregular & $\begin{array}{c}\text { Penta-, } \\
\text { hexagonal }\end{array}$ & Raised & Straight & Thin & Striate & Concave & Striate \\
\hline $\begin{array}{l}\text { 19-Origanum syriacum L. subsp. } \\
\text { sinaicum (Boiss.) Greuter\&Burdet }\end{array}$ & Ruminate & Irregular & Irregular & Raised & Undulate & Thick & Undulate & Slightly concave & Smooth \\
\hline 20-Salvia aegyptiaca $\mathrm{L}$ & Reticulate & Irregular & $\begin{array}{c}\text { Penta- } \\
\text { hexagonal }\end{array}$ & Raised & Straight & Thin & Striate & Concave & Striate \\
\hline 21-Salvia deserti Decne. & Reticulate & Irregular & $\begin{array}{c}\text { Penta- } \\
\text { hexagonal }\end{array}$ & Raised & Straight & Thin & Striate & Concave & Striate \\
\hline 22-Salvia lanigera Poir. & $\begin{array}{l}\text { Reticulate- } \\
\text { verrucate }\end{array}$ & Irregular & $\begin{array}{c}\text { Penta -, } \\
\text { hexagonal }\end{array}$ & Raised & $\begin{array}{c} \pm \\
\text { Straight } \\
\end{array}$ & \pm Thick & Striate & Concave & Favulariate \\
\hline 23-Salvia multicaulis Vahl & $\begin{array}{l}\text { Reticulate- } \\
\text { foveate }\end{array}$ & Irregular & $\begin{array}{c}\text { Penta-, } \\
\text { hexagonal }\end{array}$ & Raised & Straight & Thin & Striate & Concave & Striate \\
\hline 24-Salvia palaestina Benth. & $\begin{array}{c}\text { Smooth- } \\
\text { slightly } \\
\text { tuberculate }\end{array}$ & Irregular & $\begin{array}{c}\text { Penta-, } \\
\text { hexagonal, } \\
\text { elongated }\end{array}$ & $\begin{array}{c}\text { Slightly } \\
\text { raised }\end{array}$ & Straight & Thick & Striate & Slightly concave & Smooth \\
\hline 25-Salvia sclarea $\mathrm{L}$. & $\begin{array}{l}\text { Sclariform- } \\
\text { rugose }\end{array}$ & Irregular & $\begin{array}{c}\text { Penta-, } \\
\text { hexagonal, } \\
\text { elongated }\end{array}$ & $\begin{array}{l}\text { Slightly } \\
\text { raised }\end{array}$ & Undulate & Thick & Ribbed & $\begin{array}{l}\text { Flat - slightly } \\
\text { concave }\end{array}$ & Ruminate \\
\hline 26-Salvia spinosa $\mathrm{L}$. & $\begin{array}{l}\text { Finely } \\
\text { reticulate- } \\
\text { foveate } \\
\end{array}$ & Irregular & $\begin{array}{c}\text { Penta-, } \\
\text { hexagonal }\end{array}$ & $\begin{array}{l}\text { Slightly } \\
\text { raised }\end{array}$ & Straight & Thick & Smooth & Slightly concave & Foveate-smooth \\
\hline 27-Thymus bovei Benth. & Reticulate & Regular & $\begin{array}{l}\text { Tetra-, penta-, } \\
\text { hexagonal }\end{array}$ & Channelled & Straight & Thin & Striate & $\begin{array}{c}\text { Flat -slightly } \\
\text { concave }\end{array}$ & $\begin{array}{l}\text { Striate to micro- } \\
\text { reticulate }\end{array}$ \\
\hline 28-Thymus decussatus Benth. & $\begin{array}{c}\text { Indistict } \\
\text { Reticulate }\end{array}$ & Regular & $\begin{array}{l}\text { Tetra-, penta-, } \\
\text { hexagonal }\end{array}$ & Channelled & Undulate & Thin & Striate & $\begin{array}{l}\text { Flat -slightly } \\
\text { convex }\end{array}$ & $\begin{array}{l}\text { Striate to micro- } \\
\text { reticulate }\end{array}$ \\
\hline
\end{tabular}


Table 4. Hilum features of studied taxa.

\begin{tabular}{|c|c|c|c|c|}
\hline \multirow{2}{*}{ Taxa } & \multicolumn{4}{|c|}{ Hilum Character } \\
\hline & Shape & $\mathbf{L} \mathbf{X ~ W}(\mathbf{m m})$ & Position & Rim \\
\hline $\begin{array}{l}\text { Subfamily: Ajugoideae } \\
\text { 1- Ajuga chamaepitys (L.) Schreb. subsp. tridactylites (Benth.) P.H.Davis }\end{array}$ & Obovoid & $1.3-1.4 \times 0.6-0.7$ & Baso-lateral & + \\
\hline 2- Ajuga iva (L.) Schreb. & Obovoid & $1.8-1.9 \times 1.2-1.3$ & Sub baso-lateral & + \\
\hline 3- Teucrium decaisnei C.Presl & Globose & $1.1-1.2 \times 1-1.1$ & Sub baso-lateral & + \\
\hline 4- Teucrium leucocladum Boiss. & Subglobose- globose & $0.78-0.82 \times 0.77-0.81$ & Sub baso-lateral & + \\
\hline 5- Teucrium polium L. & Subglobose- globose & $1-1.04 \times 0.98-1.0$ & Sub baso- lateral & + \\
\hline $\begin{array}{l}\text { Subfamily: Lamioideae } \\
\text { 6-Ballota undulata (Fresen.) Benth. }\end{array}$ & Ovoid- globose or ovoid & $0.2-0.3 \times 0.2-0.28$ & Basal & - \\
\hline 7-Lamium amplexicaule L. & Triangular & $0.15-0.2 \times 0.3-0.4$ & Basal - sub basal & - \\
\hline 8-Marrubium alysson $\mathrm{L}$. & Triangular & $0.3-0.4$ X0.3 & Basal & - \\
\hline 9-Marrubium vulgare $\mathrm{L}$. & Obovoid & $0.2-0.3 \times 0.15-2$ & Basal & - \\
\hline 10-Otostegia fruticosa (Forssk.) Penz. & Triangular & $0.17-0.2 \times 0.35-0.4$ & Basal & - \\
\hline 11-Phlomis aurea Decne. & Ovoid- triangular & $0.3-0.4 \times 0.6-0.7$ & Basal & - \\
\hline 12- Stachys aegyptiaca Pers. & Quadrangular, broad triangular & $0.3-0.35 \times 0.35-0.4$ & Basal & - \\
\hline $\begin{array}{l}\text { Subfamily: Nepetoideae } \\
\text { 13-Lavandula coronopifolia } \text { Poir. }\end{array}$ & Broad obcordate- rounded & $0.4-0.5 \times 0.5-0.6$ & Basal, white & - \\
\hline 14-Lavandula pubescens Decne. & Broad obcordate- rounded & $0.5-0.6 \times 0.4-0.6$ & Basal, white & - \\
\hline 15-Mentha longifolia (L.) Huds.subsp. typhoides (Briq.)Harley & Bilobed & $0.12-0.2 \times 0.3-0.4$ & Basal, white & - \\
\hline 16-Micromeria nervosa (Desf.) Benth. & Ovoid & 0.06-0.1 X 0.05-0.1 & Basal, whitish in colour & - \\
\hline 17-Micromeria sinaica Benth. & Ellipsoid & $0.08-0.1 X 0.05-0.07$ & Basal, whitish in colour & - \\
\hline 18-Nepeta septemcrenata Benth. & Bilobed & $0.25-0.3 \times 0.6-0.8$ & Sub basal, whitish in colour & - \\
\hline 19-Origanum syriacum L. subsp. sinaicum (Boiss.) Greuter\&Burdet & \pm Globose & $0.15-0.2 \times 0.2-0.25$ & Sub basal, whitish in colour & - \\
\hline 20-Salvia aegyptiaca $\mathrm{L}$ & Ellipsoid -obovoid & $0.1-0.2 \times 0.05-0.1$ & Sub basal & - \\
\hline 21-Salvia deserti Decne. & Ellipsoid-obovoid & $0.15-0.23 \times 0.1-0.13$ & Sub basal & - \\
\hline 22-Salvia lanigera Poir. & Rhomboidal- globose & $0.25-0.35 \times 0.2-0.3$ & Sub basal, whitish in colour & + \\
\hline 23-Salvia multicaulis Vahl & Discoid & $0.35-0.4 \times 0.6-0.7$ & Basal & - \\
\hline 24-Salvia palaestina Benth. & \pm Globose & $0.13-0.16 \times 0.13-0.16$ & Basal & - \\
\hline 25-Salvia sclarea $\mathrm{L}$. & Discoid & $0.12-0.15 \times 0.2-0.3$ & Basal & - \\
\hline 26-Salvia spinosa L. & Ovoid - oblong & $0.1-0.2 \times 0.2-0.25$ & Basal, marginal, white & - \\
\hline 27-Thymus bovei Benth. & Obovoid & $0.15-0.18 \times 0.13-0.15$ & Basal, whitish in colour & - \\
\hline 28-Thymus decussatus Benth. & Obovoid & $0.12-0.15$ X $0.08-0.1$ & Basal, whitish in colour & - \\
\hline
\end{tabular}




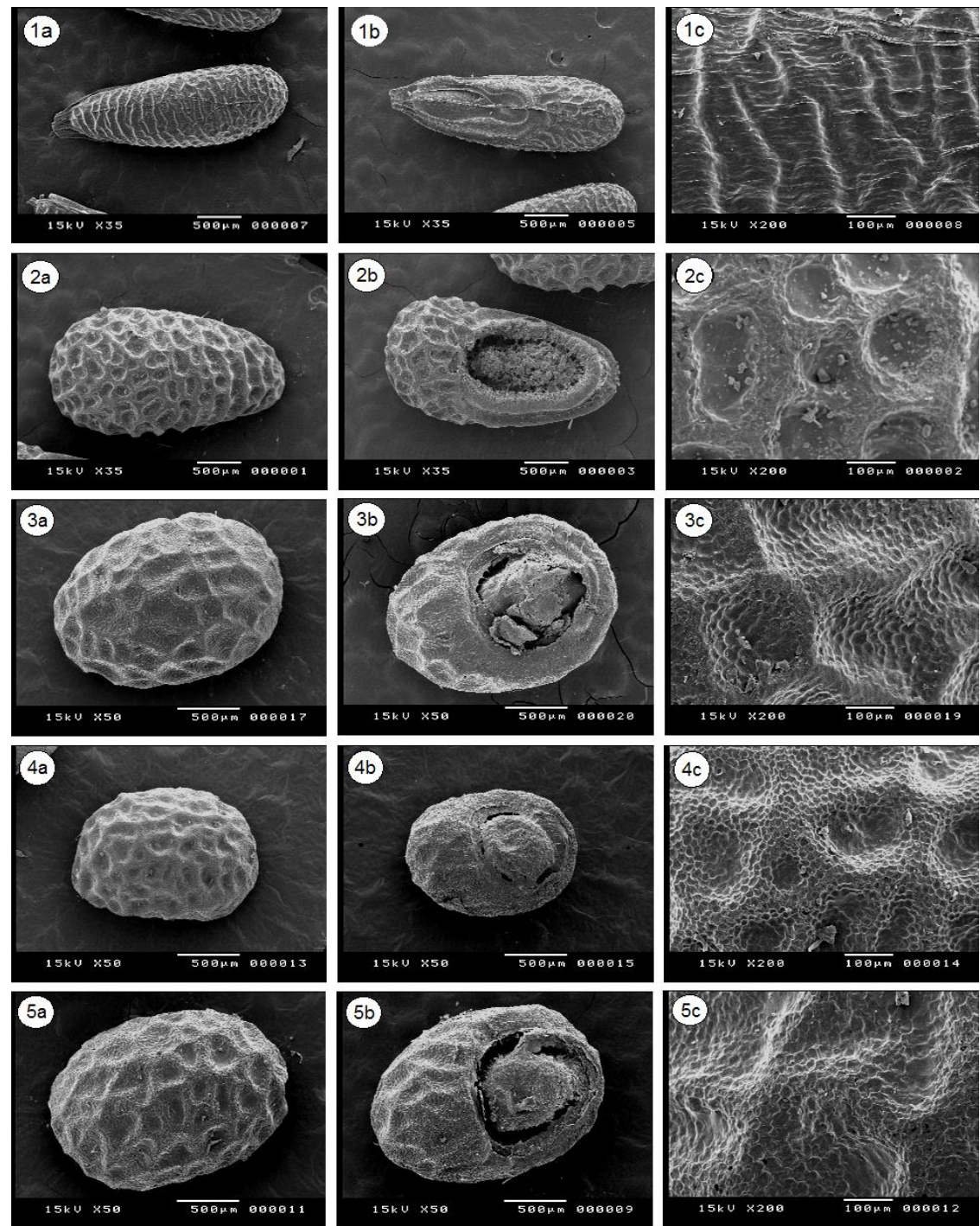

Fig. 1-5. Scanning electron micrographs of nutlets. a. dorsal view, b. ventral view, c. surface sculpture. 1. Ajuga chamaepitys. subsp. tridactylites; 2. A. iva; 3. Teucrium decaisnei; 4. T. leucocladum; 5. T. polium.

Received 17 Oct. 2014, Accepted 10 Nov. 2014 